\title{
Adult age differences in interference from a prospective- memory task: a diffusion model analysis
}

\author{
Sebastian S. Horn • Ute J. Bayen • Rebekah E. Smith
}

Published online: 25 May 2013

(C) Psychonomic Society, Inc. 2013

\begin{abstract}
People often slow down their ongoing activities when they must remember an intended action, known as the cost or interference effect of prospective memory (PM). Only a few studies have examined adult age differences in PM interference, and the specific reasons underlying such differences are not well understood. The authors used a modelbased approach to reveal processes underlying PM interference and age differences in these processes. Older and younger adults first performed a block of an ongoing lexical decision task alone. An embedded event-based PM task was added in a second block. Simultaneously accounting for the changes in response time distributions and error rates induced by the PM task, Ratcliff's (Psychological Review 85:59-108, 1978) diffusion model was used to decompose the nonlinear combination of speed and accuracy into psychologically meaningful components. Remembering an intention not only reduced processing efficiency in both age groups, but also prolonged peripheral nondecision times and induced response cautiousness. Overall, the findings suggest that there are multiple, but qualitatively similar factors underlying PM task interference in both age groups.
\end{abstract}

Keywords Aging · Diffusion model · Prospective memory · Monitoring and attentional resources $\cdot$ Older adults

Electronic supplementary material The online version of this article (doi:10.3758/s13423-013-0451-y) contains supplementary material, which is available to authorized users.

S. S. Horn · U. J. Bayen

Heinrich-Heine-Universität,

Düsseldorf, Germany

S. S. Horn $(\bowtie)$

Max Planck Institute for Human Development,

Berlin, Germany

e-mail: Sebastian.Horn@mpib-berlin.mpg.de

R. E. Smith

The University of Texas at

San Antonio, San Antonio, TX, USA
Prospective memory (PM) involves remembering intended actions after a delay, such as remembering to buy groceries after work. PM is essential for an independent lifestyle, particularly in old age (e.g., Schnitzspahn, Ihle, Henry, Rendell, \& Kliegel, 2011). PM tasks involve the retrieval of information from the past (the retrospective component) and also require us to remember that there was something we intended to do (prospective component). Research on PM has largely focused on how intentions come into mind at the relevant moment and whether this process requires attentional resources. To address these questions in the laboratory, the PM task and target events (e.g., "remember to press the space bar when you see the word tiger") are embedded in an ongoing task (e.g., a lexical decision task [LDT]). One fairly pervasive finding is the cost or interference effect of PM (Smith, 2003). That is, response times (RTs) on nontarget trials of the ongoing task are often increased in the presence of a PM task, relative to performing the ongoing task alone. It has been proposed that interference indicates resources being devoted to monitoring (e.g., Guynn, 2003) or for preparatory attentional processes (Smith, 2003). Although there is debate as to whether these processes are necessary for all PM tasks, theoretical views concur that the prospective component involves resource-demanding processes leading to interference effects in nonfocal tasks. Nonfocal tasks require a shift from the processing routine during the ongoing task toward the relevant PM target features (see McDaniel, Einstein, \& Rendell, 2008, for details) and show reliable age-related PM differences favoring young adults (e.g., Henry, MacLeod, Phillips, \& Crawford, 2004). In the present study, we investigated the cognitive processes underlying task interference and how these processes may vary in young and older adults.

Although interference is an important indicator of allocation of attention toward the PM task, the specific processes giving rise to the slowing are not well understood. Thus, one important research goal is to identify processes that 
contribute to PM task interference. Moreover, a limitation to previous analyses of age-related differences in PM interference stems from scaling dependency and unequal baseline performance between young and older adults (Perfect \& Maylor, 2000). That is, conclusions about task-induced changes in RTs between age groups (treatment $\times$ age interactions) are not meaningful if the assumption of a common interval scale across age groups is violated, if the relationship between latent processes and RT is unclear, or if the nonlinearity of speed-accuracy functions is ignored (see Verhaeghen, 2000; Wagenmakers, Krypotos, Criss, \& Iverson, 2012).

We addressed these issues by applying the diffusion model that explicitly accounts for the nonlinear combination of speed and accuracy and does not treat RT as a process-pure variable. Prior applications of the model have shown that participants' speed-accuracy criteria often differ as a function of age, with older adults sacrificing speed for accuracy (e.g., Ratcliff, Thapar, Gomez, \& McKoon, 2004). We therefore expected longer RTs and higher LDT accuracy for older relative to young adults. We also included a PM task embedded in the LDT and, on the basis of prior research (e.g., Gao, Cheung, Chan, Chu, \& Lee, 2013; McDaniel et al., 2008; Smith \& Bayen, 2006), we expected PM interference for both age groups. Our goal was to model whether the same or qualitatively different processes are responsible for PM interference in young and older adults, to determine whether there are agerelated differences in the size of these effects, and to confirm findings from previous applications of the diffusion model in PM studies with younger adults.

\section{The diffusion model}

The diffusion model (Ratcliff, 1978) has been successfully applied to many speeded two-choice tasks and has contributed to our understanding of the processes underlying agerelated slowing in lexical decisions (Ratcliff et al., 2004), signal detection, and recognition memory (Ratcliff, Thapar, $\&$ McKoon, 2006), among others. By decomposing accuracy and the whole RT distributions for correct and error responses into psychologically substantive components, the model provides a comprehensive and detailed approach to examining why RTs are long or short and why few or many errors are made. For instance, the hypothesis of a decrease in processing speed with aging (Salthouse, 1996) was refined by showing that there are separable ways that such slowing can be produced. As one major finding, older adults set more conservative speed-accuracy criteria and have longer nondecision times (including motor execution) across many tasks (e.g., Ratcliff et al., 2004; Ratcliff et al., 2006). Across many applications, the diffusion model has provided a close fit to observed data, and the discriminant validity of its parameters has been demonstrated (Voss, Rothermund, \& Voss, 2004).

\section{Model parameters}

One core model assumption is that decisions are based on continuous information accumulation over time, defined by a Wiener diffusion process moving from a starting point until one of two decision boundaries is crossed (Fig. 1).

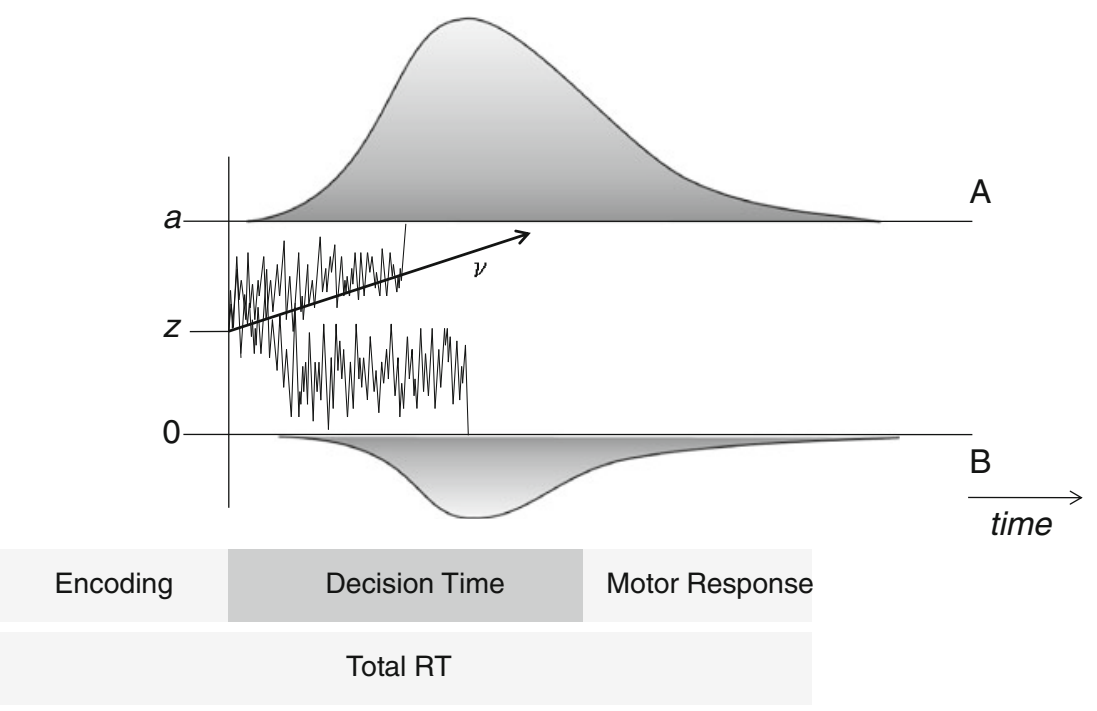

Fig. 1 Illustration of the diffusion model (Ratcliff, 1978). The vertical axis is the decision-related strength-of-evidence axis, and the horizontal axis is the time axis. Diffusion processes start at point $z$ and move over time until the upper boundary (positioned at $a$ ) or the lower boundary (positioned at zero) is reached. Decision time distributions associated with the upper and the lower boundaries are shown. The two oscillating sample tracks illustrate that different boundaries can be reached with the same (positive) drift rate due to random influence, as given by the diffusion constant $s$ (which is set to 1 in all present analyses). Total response time (RT) is the sum of the decision time and a nondecision component $T_{\mathrm{er}}$ that represents the duration of processes such as encoding and motor response execution 
Accumulation within a trial is noisy, implying that diffusion processes for equivalent items will not deterministically reach the same boundary at the same time and will, thus, produce errors and RT distributions. The model version we used includes the following parameters.

\section{Mean drift rate $\nu$}

This parameter quantifies the average speed of information uptake. Drift rate represents processing efficiency, with higher absolute values leading to both faster and more accurate decisions, thus reflecting participant ability or task difficulty.

\section{Boundary separation a}

The distance $a$ between the two decision boundaries quantifies the amount of evidence required until a decision is made, representing the speed-accuracy setting. By increasing $a$, the decision maker can trade speed for accuracy (a more "cautious" setting), or vice versa.

\section{Mean nondecision time $T_{\text {er }}$}

Observed RT can be additively split into decision and nondecision time (i.e., $\mathrm{RT}=\mathrm{DT}+T_{\mathrm{er}}$ ). The latter includes the processes before and after the actual decision phase (such as encoding and motor execution). Changes in $T_{\text {er }}$ imply shifts of the entire RT distribution without changing accuracy.

\section{Variability parameters $\eta, s_{z}, s_{t}$}

The model assumes variation of some parameters across trials. Drift rate varies normally around mean $v$ with standard deviation $\eta$. The starting point and nondecision time are uniformly distributed around mean $z$ and $T_{\mathrm{er}}$, with ranges $s_{\mathrm{z}}$ and $s_{\mathrm{t}}$, respectively. With intertrial variability of drift rate $\eta$, the model can account for longer RTs for incorrect responses than for correct responses, and variability in the starting point $\left(s_{\mathrm{z}}\right)$ can account for a reverse pattern (e.g., Wagenmakers, Ratcliff, Gomez, \& McKoon, 2008).

Modeling ongoing-task performance in the PM paradigm

Although various ongoing tasks used in PM research could provide appropriate data for application of the model, we used an LDT, since it has been studied in detail with the diffusion model (see Ratcliff et al., 2004; Wagenmakers et al., 2008). Only a few PM studies have applied the model, and these were exclusively with young adults (Boywitt \& Rummel, 2012; Horn, Bayen, \& Smith, 2011; Horn, Smith, Bayen, \& Voss, 2008). In the present study, we focused on age comparisons in processes underlying PM task interference. From results with younger adults, we predicted that
PM tasks induce cautiousness (boundary-separation parameter $a$ ), reflecting a shift in the approach to the ongoing task (Horn et al., 2008). Moreover, we assumed that the time before or after an ongoing-task decision (the nondecision-time parameter $T_{\mathrm{er}}$ ) is prolonged if participants strategically check for the presence of PM targets (Horn et al., 2011).

\section{Method}

Participants

Forty-six young adults (11 male; $M=13.51$ years of education) and 43 community-dwelling older adults (18 male; $M=11.95$ years of education) participated. The participants, all native German speakers, were recruited through newspaper advertisements or a department pool and received money or psychology course credit. Individuals with major health problems that may affect cognitive functioning were excluded. Older adults scored higher than young adults on verbalsemantic knowledge, but lower in the digit-symbol test and short-term memory span (details in Table 1).

Table 1 Participant characteristics, cognitive tests, and memory performance

\begin{tabular}{|c|c|c|c|c|c|}
\hline \multirow[t]{2}{*}{ Variable } & \multicolumn{2}{|c|}{ Young Adults } & \multicolumn{2}{|c|}{ Older Adults } & \multirow{2}{*}{$\eta_{\mathrm{p}}^{2}$} \\
\hline & $M$ & $S D$ & $M$ & $S D$ & \\
\hline Age & 23.0 & 3.5 & 70.0 & 5.0 & \\
\hline Vocabulary $^{\mathrm{a}}$ & 32.2 & 2.2 & 33.1 & 1.5 & $.05 *$ \\
\hline Processing speed ${ }^{\text {a }}$ & 88.3 & 13.4 & 61.8 & 11.9 & $.53 * * *$ \\
\hline Digit span ${ }^{a}$ & 19.6 & 3.4 & 16.3 & 3.2 & $.20 * * *$ \\
\hline Prospective memory ${ }^{b}$ & .63 & .27 & .44 & .31 & $.11 *$ \\
\hline Posttask recognition ${ }^{c}$ & .99 & .05 & .83 & .33 & $.11 * *$ \\
\hline
\end{tabular}

$\eta_{\mathrm{p}}^{2}=$ size of age effect

a Raw test scores. Vocabulary $=$ multiple-choice vocabulary test (MWT-A; Lehrl, Merz, Burkhard, \& Fischer, 1991). Processing speed (digit-symbol substitution) and digit span (combined forward/backward span) were measured with the German version of the Wechsler Adult Intelligence Scale (WAIS-III; von Aster, Neubauer, \& Horn, 2006)

${ }^{\mathrm{b}}$ Prospective memory (PM groups only) was measured as the proportion of target events to which participants made a correct PM response (i.e., a space bar press on a PM target or within the subsequent two LDT trials)

${ }^{\mathrm{c}}$ Posttask recognition of letters was measured as hit rate minus false alarm rate and is collapsed across the PM and no-PM groups in Table 1. Recognition did not differ between experimental groups, $F(1,85)=$ 1.02 , within younger $(M=1$ vs. $M=.99)$ or older $(M=.87$ vs. $M=.79)$ adults

$* p<.05$

$* * p<.01$

$* * * p<.001$ 


\section{Design}

Participants were randomly assigned either to the PM group (22 young, 21 older), which first performed a baseline block of the LDT alone, followed by a second block with an embedded event-based PM task, or to the no-PM control group (24 young, 22 older), which performed the LDT alone in both blocks. The design thus included the factors age (young vs. older), experimental group (PM vs. control), and block (first vs. second).

\section{Materials}

We selected 252 low-frequency $(M=4.46$ occurrences per million, $S D=0.50)$ and 252 very-low-frequency German words $(M=0.35, S D=0.48)$ from the CELEX lexical database. Word length ranged from 8 to 12 letters $(M=$ $9.88, S D=1.27)$. Pronounceable pseudowords were generated from each word by randomly replacing one interior vowel/umlaut with a different vowel/umlaut. From this pool, we created two nontarget sets for the ongoing LDT and two target sets for the PM task, matched for word frequency and length. Each nontarget set contained three stimulus types: 120 low-frequency words, 120 very-low-frequency words, and 240 pseudowords (created from the words of the other nontarget set). The two target sets each contained 6 words and 6 pseudowords with initial letters $G, H$, or $M$, occurring equally often within a target set. None of the nontarget items started with these letters. During the experiment, the program selected one filler and one target set, from which items were randomly drawn without replacement, under the restriction of equal numbers of words and pseudowords and of lowfrequency and very-low-frequency words within each block. The possible combinations of target and distractor sets were counterbalanced across participants and conditions.

\section{Procedure}

Participants completed 24 practice trials, followed by two blocks of 252 LDT trials (50\% words) each. Each trial started with a fixation cross that remained in the center of the screen for 400, 450, 500, 550, or $600 \mathrm{~ms}$, with fixation display times randomly selected with equal probability to prevent anticipatory responding. Subsequently, a string appeared in black uppercase letters (sans-serif font, 24 point) in the center of a white screen. Participants were asked to categorize each string as a word or nonword as quickly and accurately as possible by pressing the $J$ - or F-key. Key-response mappings were counterbalanced across participants. A blank screen followed each response for $200 \mathrm{~ms}$.

After the first block, participants were informed that they should memorize some letters. Participants in the PM group were then asked to remember to press the space bar (instead of the $F$ or $J$ key) if a string started with one of the letters $G$, $H$, or $M$ in the subsequent block. In both groups, participants were informed that their memory for these letters would be tested later, and the letters were then presented in random order for $5 \mathrm{~s}$ each. Participants reproduced these letters by typing them into the keyboard. If mistakes occurred, the letters were again displayed until perfect recall. The experiment then continued with a filler digit-span task $(2 \mathrm{~min})$, followed by the second block. There was no further mention of the PM task.

The 12 PM target events appeared within trial ranges (starting from trials 17-20), with 17-23 nontarget trials between any two targets. Finally, participants completed a questionnaire, cognitive tests, and an old-new recognition task in which the three target letters and three lures appeared one at a time in random order. Sessions lasted about $1 \mathrm{~h}$.

\section{Results}

Prospective memory and target recognition

Young adults showed higher PM performance than did older adults (concurring with many laboratory studies; Henry et al., 2004) and posttask recognition of target letters (Table 1).

\section{Ongoing-task performance}

For analyses of ongoing-task performance, as is standard in PM research, we excluded PM responses (hits, false alarms) and the two trials following these responses (e.g., Smith \& Bayen, 2006). Applying the same cutoffs for the LDT as Ratcliff et al. (2004), we removed RTs smaller than $350 \mathrm{~ms}$ or greater than $4,000 \mathrm{~ms}$ for older adults $(1.1 \%$ of the data) and RTs smaller than $300 \mathrm{~ms}$ or greater than $3,000 \mathrm{~ms}$ for young adults ( $0.9 \%$ of the data). Table 2 shows descriptive statistics.

\section{Baseline performance}

Older adults' lexical decisions in block 1 were slower, $F(1$, $85)=35.73, \eta_{\mathrm{p}}{ }^{2}=.30, p<.01$, but more accurate, $F(1,85)=$ $38.15, \eta_{\mathrm{p}}{ }^{2}=.31, p<.01$, than those of young adults. As was expected for the baseline block, in which all participants worked on the ongoing LDT only, there were no differences (nor interactions) involving the experimental groups (PM, no-PM), $F \mathrm{~s}<1$.

\section{Effects of the PM task}

Both younger and older adults' RTs increased substantially with an embedded PM task in the second block (see 
Table 2 Response times and accuracy in the ongoing lexical decision task

\begin{tabular}{|c|c|c|c|c|c|c|}
\hline \multirow[t]{2}{*}{ Variable } & \multirow[t]{2}{*}{ Group } & \multicolumn{2}{|c|}{ Block 1} & \multicolumn{2}{|c|}{ Block 2} & \multirow[t]{2}{*}{$\eta_{\mathrm{p}}{ }^{2}$} \\
\hline & & $M$ & $(S E)$ & $M$ & $(S E)$ & \\
\hline \multirow[t]{6}{*}{ RT } & Young & & & & & \\
\hline & PM & 980 & (63) & 1,199 & $(70)$ & $.66 * * *$ \\
\hline & No-PM control & 931 & $(60)$ & 902 & $(67)$ & $.18 *$ \\
\hline & Older & & & & & \\
\hline & PM & 1,312 & $(65)$ & 1,693 & $(71)$ & $.63 * * *$ \\
\hline & No-PM control & 1,350 & $(63)$ & 1,296 & $(70)$ & $.20 *$ \\
\hline \multirow[t]{6}{*}{ Accuracy } & Young & & & & & \\
\hline & PM & .91 & $(.01)$ & .93 & $(.01)$ & $.28 * *$ \\
\hline & No-PM control & .90 & $(.01)$ & .90 & $(.01)$ & .02 \\
\hline & Older & & & & & \\
\hline & PM & .95 & $(.01)$ & .95 & $(.01)$ & .04 \\
\hline & No-PM control & .96 & $(.01)$ & .95 & $(.01)$ & .08 \\
\hline
\end{tabular}

The PM groups received instructions for the PM task after block 1. RT = response time (in milliseconds); accuracy = proportion of correct lexical decisions; $\eta_{\mathrm{p}}{ }^{2}=$ effect size for within-subjects change block 2-block 1

$* p<.05$

$* * p<.01$

$* * * p<.001$

Table 2), and this interference effect was larger for older adults. ${ }^{1}$ Moreover, the slowing was positively correlated with PM performance in young, $r=.66, p<.01$, and older, $r=.86, p<.01$, adults.

Variability in accuracy is usually smaller in the LDT than is variability in latencies (e.g., Ratcliff et al., 2004). Older adults' accuracy was very high and not affected by PM instructions. Yet we observed a statistically reliable increase in young adults' accuracy with a PM task. These results emphasize the importance of simultaneously modeling speed-accuracy changes.

\section{Diffusion model analysis}

\section{Parameter estimation and model fit}

We fit diffusion models separately to the data from each participant and block. For parameter estimation, we used the fast-dm method, which minimizes the maximal vertical distance between the observed and predicted cumulative RT distributions (Voss \& Voss, 2008). This estimation method is

\footnotetext{
${ }^{1}$ The supplementary materials include comprehensive three-factorial ANOVA tables. For RTs, follow-up analyses within the PM group indicated a significant two-way age $\times$ block interaction, $F(1,41)=$ $5.05, \eta_{\mathrm{p}}{ }^{2}=.11, p=.03$, suggesting that older adults were disproportionately slowed by the PM task. However, as was noted in the introduction, it is preferable to address the potential problem of scaledependent ("removable") interactions in RTs by focusing on model parameters (Wagenmakers et al., 2012).
}

relatively robust against outliers and provides a useful option if trial numbers are suboptimal for quantile-based categorization of RT distributions (we also tried other estimation approaches, which yielded similar outcomes; Vandekerckhove, Tuerlinckx, \& Lee, 2011). ${ }^{2}$ To examine the impact of our PM manipulation in this first study with older adults, all model parameters were allowed to vary between blocks, reflecting the fact that no data are as of yet available that would allow strong a priori constraints for the PM paradigm. In addition, drift rates were allowed to vary between stimulus types. Overall, the models provided good individual fits to the data from older and young adults, as indicated by fit index $p$ (the probability value of the Kolmogorov-Smirnov statistic under the null hypothesis of perfect fit). The supplemental materials include further details about model fitting and estimates of variability parameters.

\section{Modeling results: baseline}

We first examined baseline performance (block 1) between age and experimental groups. Figure 2 shows the distributions of best-fitting parameters. Drift rate $^{3}$ did not differ between

\footnotetext{
${ }^{2}$ Although Bayesian hierarchical parameter estimation would be a very fruitful approach for future aging studies, we did not apply this method here because numerical problems caused slow convergence for some participants.

${ }^{3}$ We report (absolute) drift rates averaged across stimulus types, since lexical variables were not the focus of the study. Supplement 3 includes the estimates as a function of stimulus type, replicating typical effects of word frequency (cf. Ratcliff et al., 2004).
} 

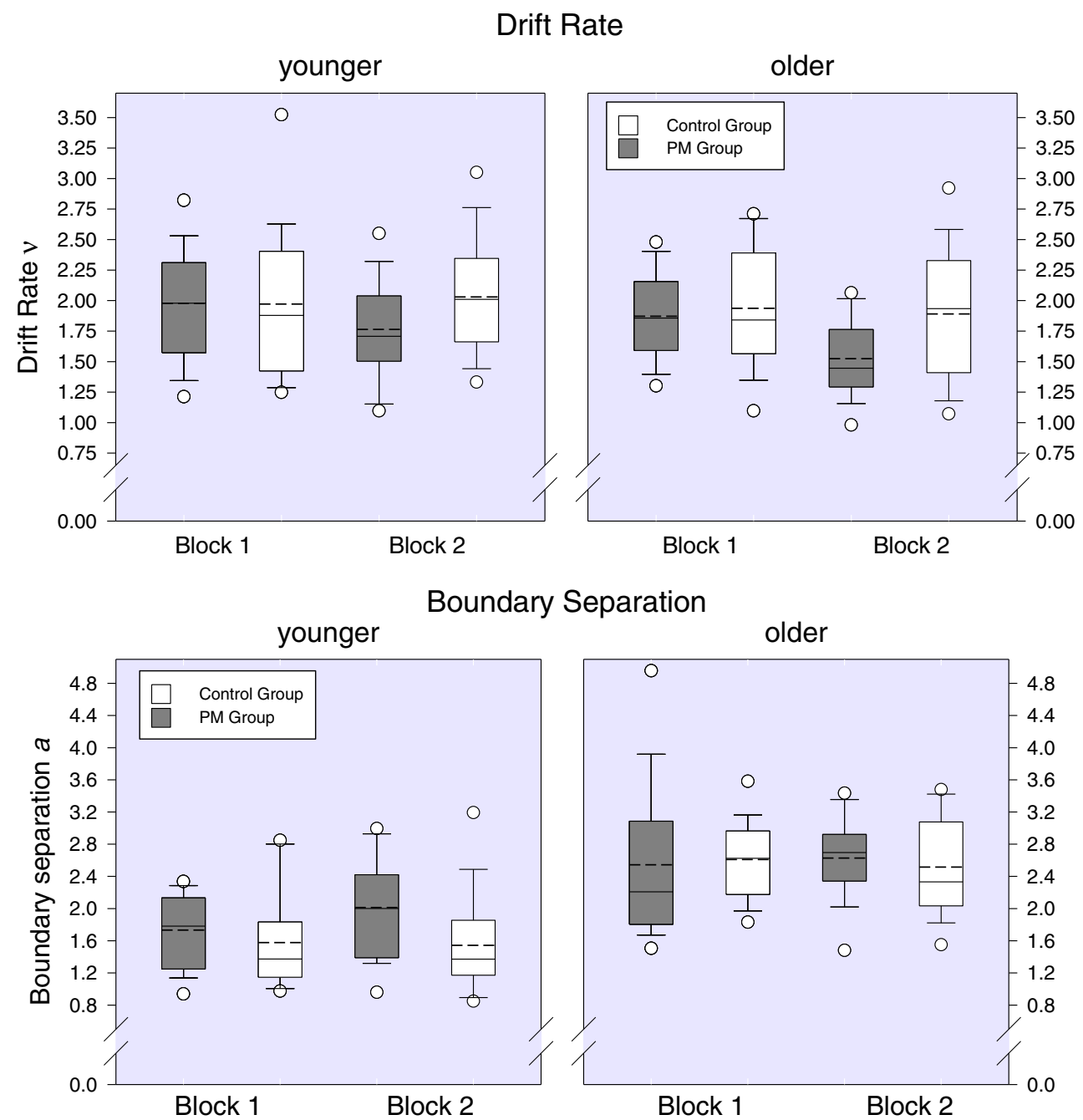

Nondecision Time
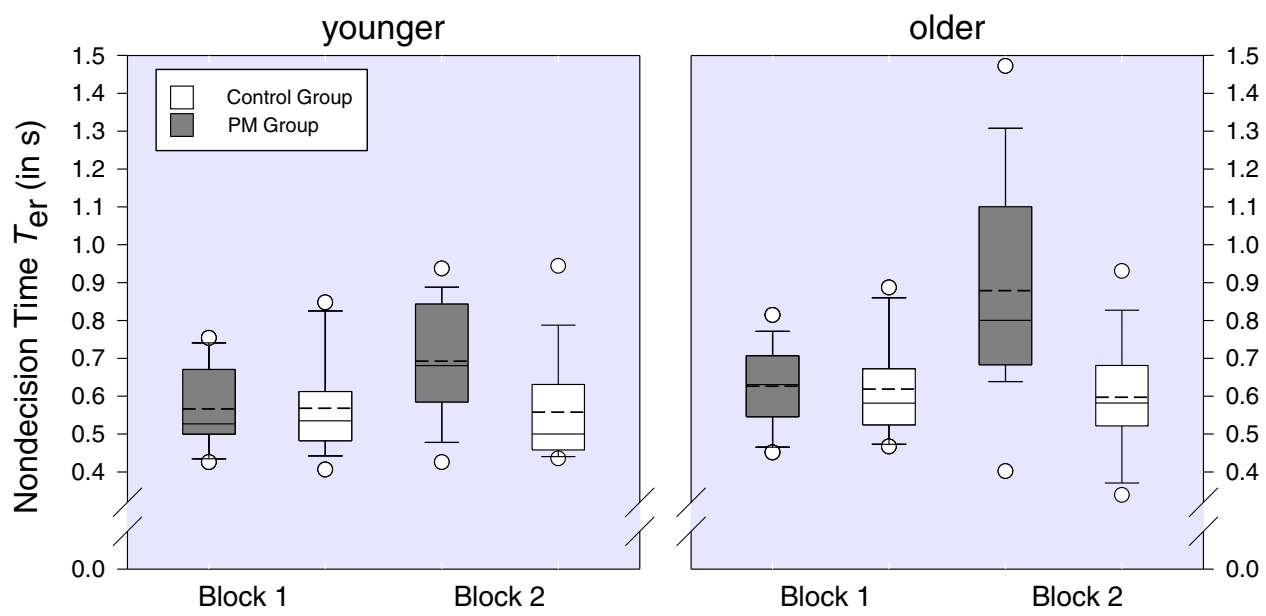

Fig. 2 Best-fitting diffusion model parameters. Drift rate $\nu$, boundary separation $a$, and nondecision time $T_{\mathrm{er}}$ as a function of age (young vs. older), experimental group (PM, no-PM control), and medians (solid lines) and means (dashed lines). Whiskers (error bars) below/above the boxes mark the 10th and 90th percentiles, and dots mark the 5th and 95th percentiles. Scaling parameter block (first vs. second). The box-and-whisker plots include the (diffusion constant) $s=1$ 
age groups, $F<1$. However, older adults were substantially more cautious (increased boundary separation), $F(1,85)=$ $48.06, \eta_{\mathrm{p}}{ }^{2}=.36, p<.01$, and their nondecision times were longer than those of young adults, $F(1,85)=4.91, \eta_{\mathrm{p}}{ }^{2}=.06$, $p<.05$. These results are consistent with previous research (Ratcliff et al., 2004), showing that the main source underlying adult-age differences in LDTs are not the drift rates and reveal that older adults were slower but more accurate due to more conservative speed-accuracy settings. As was expected for the baseline block, there were no main effects (nor interactions) involving the experimental groups (all $F_{\mathrm{S}}<1$ ).

\section{$P M$ and drift rate}

We analyzed the change in parameters of main interest between the baseline and PM blocks within participants. Both older and younger adults' drift rates decreased with an embedded PM task, $F(1,41)=22.37, \eta_{\mathrm{p}}{ }^{2}=.35, p<.01$, and there was no differential impact of age. This suggests that PM interfered with the efficiency of processing the letter strings, since individuals may be forced to trade off their limited attentional resources between the ongoing and the PM task. In the control group, younger and older adults' drift rates did not change between blocks, $F<1{ }^{4}$

\section{PM and boundary separation a}

In line with previous findings (e.g., Horn et al., 2008), boundary separation tended to increase after assignment of the PM task, $F(1,41)=3.23, \eta_{\mathrm{p}}{ }^{2}=.07, p=.08$; that is, participants in the PM group responded more cautiously and required more information for ongoing decisions, ${ }^{5}$ relative to participants in the control group (as reflected by the significant block $\times$ group interaction; Supplement 1). There is evidence that participants perceive ongoing decisions as more complex with embedded PM tasks, inducing criterion shifts to respond more cautiously to detect targets (Boywitt \& Rummel, 2012). No criterion changes between blocks were observed in the control groups, $F(1,44)=1.38, p=.25$.

\section{$P M$ and nondecision time $T_{e r}$}

Peripheral nondecision time increased substantially with an additional PM task, $F(1,41)=49.05, \eta_{\mathrm{p}}{ }^{2}=.55, p<.01$. Notably, this effect was larger for older than for younger

\footnotetext{
$\overline{{ }^{4} \text { Reflecting PM }}$ task interference, experimental group $\times$ block interactions emerged across all model parameters and RTs (see Supplement 1). Consequently, we report follow-up analyses separately for the PM and control groups.

${ }^{5}$ Separate tests within the PM group indicate strong increases in younger adults' boundary separation, $t(21)=3.54, \eta_{\mathrm{p}}{ }^{2}=.37, p<.01$. Older adults' changes in boundary separation were not reliable, $t<1$, presumably due to generally conservative settings from the outset.
}

adults $\left[F(1,41)=5.43, \eta_{\mathrm{p}}{ }^{2}=.12, p<.05\right.$, for the age $\times$ block interaction within the PM group], suggesting that this component may be the primary source underlying the interaction found in RTs. Peripheral processes are likely prolonged if participants check whether strings are PM targets before/after their ongoing lexical decisions (see Horn et al., 2011). Also, consistent with Gao et al. (2013), this finding may point to older adults' disproportionately reduced execution speed. In the control group, no changes across blocks emerged in younger and older adults' nondecision times, $F(1,44)=1.38, p=.25$.

\section{Discussion}

In line with previous work (Ratcliff et al., 2004; Ratcliff et al., 2006), older adults' lexical decisions were slower but more accurate than those of young adults due to more conservative speed-accuracy settings and longer nondecision time. By applying the diffusion model to decompose the nonlinear combination of speed and accuracy into psychologically meaningful components (Ratcliff, 1978), we accounted for these age-related baseline differences when examining interference from a PM task, which we found for both age groups.

The embedded PM task reduced processing efficiency $(v)$ in lexical decisions in both age groups to a similar extent and tended to increase cautiousness. Thus, one portion of the slowing may be attributable to shifts in speed-accuracy criteria. The counterintuitive implication is that intervening ongoing activities may be approached more slowly but with higher accuracy if participants must remember an intention. Such effects are detectable via the diffusion model but are often masked in comparisons of average performance if accuracy is relatively high, as in the LDT (Horn et al., 2011). Notably, prolonged nondecision time was the largest contributor to PM interference. At least in demanding PM tasks (as in the present study), participants may engage in intermittent target-checking before or after their ongoing lexical decisions (see Guynn, 2003). These effects in peripheral processes were larger for older adults, pointing to disproportionately slowed or thorough checking. The results also concur with those of Gao et al. (2013), who identified lower motor execution speed as a mediator of increased PM task interference in old age.

In the present study, increases in RTs with an embedded PM task were larger for older than for younger adults (see also Gao et al., 2013; McDaniel et al., 2008). Other studies have not found age-related differences (Einstein, McDaniel, \& Scullin, 2011) or have sometimes shown a tendency toward larger interference for young adults (Smith \& Bayen, 2006). A parsimonious explanation for this apparent inconsistency is that resource allocation policies likely differ across individuals 
and tasks. In more demanding PM tasks, reduced attentional capacity may force older adults to either sacrifice ongoingtask performance for maintaining adequate levels of PM or vice versa. Consequently, age-related differences in PM performance favoring young adults were more pronounced whenever interference did not differ between age groups (Einstein et al., 2011; Smith \& Bayen, 2006).

In conclusion, the modeling revealed that the same processes contribute to PM task interference in young and older adults. The general pattern is thus consistent with aging theories assuming qualitatively common mechanisms underlying interference effects (see Verhaeghen, 2000, for a discussion of parallelisms in task interference). We believe that the diffusion-modeling approach provided insight into the PM interference effect not attainable with mere analyses of mean RT. An interesting avenue for further research may be to apply the model to examine age differences in less resource demanding PM tasks.

Author Note Sebastian S. Horn, Max Planck Institute for Human Development Berlin, Center for Adaptive Rationality (ARC). Ute J. Bayen, Institut für Experimentelle Psychologie, Heinrich-HeineUniversität Düsseldorf. Rebekah E. Smith, Department of Psychology, The University of Texas at San Antonio.

We thank Christina Engels, Maike Lex, and Sarah Müller for assistance with data collection. This project was part of Sebastian S. Horn's doctoral dissertation. Support was provided to R.E.S. by Grant AG034965 from the National Institute on Aging. Correspondence concerning this article should be addressed to Sebastian Horn, Max Planck Institute for Human Development, Center for Adaptive Rationality (ARC), Lentzeallee 94, 14195 Berlin, Germany, E-mail: Sebastian.Horn@mpib-berlin.mpg.de

\section{References}

Boywitt, C. D., \& Rummel, J. (2012). A diffusion model analysis of task interference effects in prospective memory. Memory \& Cognition, 40, 70-82.

Einstein, G. O., McDaniel, M. A., \& Scullin, M. K. (2011). Prospective memory and aging: Understanding the variability. In M. NavehBenjamin \& N. Ohta (Eds.), Memory and aging: Current issues and future directions. Philadelphia, PA: Psychology Press.

Gao, J. L., Cheung, R. T. F., Chan, Y. S., Chu, L. W., \& Lee, T. M. C. (2013). Increased prospective memory interference in normal and pathological aging: different roles of motor and verbal processing speed. Aging, Neuropsychology, and Cognition, 20, 80-100.

Guynn, M. J. (2003). A two-process model of strategic monitoring in event-based prospective memory: Activation/retrieval mode and checking. International Journal of Psychology, 38, 245-256.

Henry, J. D., MacLeod, M. S., Phillips, L. H., \& Crawford, J. R. (2004). A metaanalytic review of prospective memory and aging. Psychology and Aging, 19, 27-39.

Horn, S. S., Bayen, U. J., \& Smith, R. E. (2011). What can the diffusion model tell us about prospective memory? Canadian Journal of Experimental Psychology, 65, 69-75.
Horn, S. S., Smith, R. E., Bayen, U. J., \& Voss, A. (2008). Analyzing the cost of prospective memory with the diffusion model. International Journal of Psychology, 43, 787.

Lehrl, S., Merz, J., Burkhard, G., \& Fischer, S. (1991). MehrfachwahlWortschatz-Intelligenztest (MWT-A). Goettingen, Germany: Hogrefe.

McDaniel, M. A., Einstein, G. O., \& Rendell, P. G. (2008). The Puzzle of inconsistent age-related declines in prospective memory: A multiprocess explanation. In M. Kliegel, M. A. McDaniel, \& G. O. Einstein (Eds.), Prospective memory: Cognitive, neuroscience, developmental, and applied perspectives (pp. 141-160). Mahwah, NJ: Erlbaum.

Perfect, T. J., \& Maylor, E. A. (2000). Rejecting the dull hypothesis: The relation between method and theory in cognitive aging research. In T. J. Perfect \& E. A. Maylor (Eds.), Models of cognitive aging (pp. 1-18). Oxford: University Press.

Ratcliff, R. (1978). A theory of memory retrieval. Psychological Review, 85, 59-108.

Ratcliff, R., Thapar, A., Gomez, P., \& McKoon, G. (2004). A diffusion model analysis of the effects of aging in the lexical-decision task. Psychology and Aging, 19, 278-289.

Ratcliff, R., Thapar, A., \& McKoon, G. (2006). Aging and individual differences in rapid two-choice decisions. Psychonomic Bulletin \& Review, 13, 626-635.

Salthouse, T. A. (1996). The processing speed theory of adult age differences in cognition. Psychological Review, 103, 403428.

Schnitzspahn, K. M., Ihle, A., Henry, J. D., Rendell, P. G., \& Kliegel, M. (2011). The age-prospective memory-paradox: An exploration of possible mechanisms. International Psychogeriatrics, 23, 583592.

Smith, R. E. (2003). The cost of remembering to remember in eventbased prospective memory: Investigating the capacity demands of delayed intention performance. Journal of Experimental Psychology: Learning, Memory, and Cognition, 29, 347-361.

Smith, R. E., \& Bayen, U. J. (2006). The source of adult age differences in event-based prospective memory: A multinomial modeling approach. Journal of Experimental Psychology: Learning, Memory, and Cognition, 32, 623-635.

Vandekerckhove, J., Tuerlinckx, F., \& Lee, M. D. (2011). Hierarchical diffusion models for two-choice response times. Psychological Methods, 16, 44-62.

Verhaeghen, P. (2000). The parallels in beauty's brow: Time-accuracy functions and their implications for cognitive aging theories. In T. J. Perfect \& E. A. Maylor (Eds.), Models of cognitive aging (pp. 50-86). Oxford: University Press.

von Aster, M., Neubauer, A., \& Horn, R. (2006). Wechsler Intelligenztest für Erwachsene (WIE). Deutschsprachige Bearbeitung und Adaptation des WAIS-III von David Wechsler. Frankfurt, Germany: Pearson.

Voss, A., Rothermund, K., \& Voss, J. (2004). Interpreting the parameters of the diffusion model: An empirical validation. Memory \& Cognition, 32, 1206-1220.

Voss, A., \& Voss, J. (2008). A fast numerical algorithm for the estimation of diffusion model parameters. Journal of Mathematical Psychology, 52, 1-9.

Wagenmakers, E.-J., Krypotos, A.-M., Criss, A. H., \& Iverson, G. (2012). On the interpretation of removable interactions: A survey of the field 33 years after Loftus. Memory \& Cognition, 40, 145-160.

Wagenmakers, E. J., Ratcliff, R., Gomez, P., \& McKoon, G. (2008). A diffusion model account of criterion shifts in the lexical decision task. Journal of Memory and Language, 58, 140-159. 